\title{
Clustering Very Large Multi-dimensional Datasets with MapReduce.
}

\author{
Robson L. F. Cordeiro \\ CS Department - ICMC, \\ University of São Paulo - Brazil \\ robson@icmc.usp.br \\ Julio López \\ SCS, Carnegie Mellon \\ University - USA \\ jclopez@andrew.cmu.edu
}

\author{
Caetano Traina Jr. \\ CS Department - ICMC, \\ University of São Paulo - Brazil \\ caetano@icmc.usp.br \\ U Kang \\ SCS, Carnegie Mellon \\ University - USA \\ ukang@cs.cmu.edu
}

\author{
Agma J. M. Traina \\ CS Department - ICMC, \\ University of São Paulo - Brazil \\ agma@icmc.usp.br \\ Christos Faloutsos \\ SCS, Carnegie Mellon \\ University - USA \\ christos@cs.cmu.edu
}

\begin{abstract}
Given a very large moderate-to-high dimensionality dataset, how could one cluster its points? For datasets that don't fit even on a single disk, parallelism is a first class option. In this paper we explore MapReduce for clustering this kind of data. The main questions are (a) how to minimize the I/O cost, taking into account the already existing data partition (e.g., on disks), and (b) how to minimize the network cost among processing nodes. Either of them may be a bottleneck. Thus, we propose the Best of both Worlds - BoW method, that automatically spots the bottleneck and chooses a good strategy. Our main contributions are: (1) We propose $B o W$ and carefully derive its cost functions, which dynamically choose the best strategy; (2) We show that BoW has numerous desirable features: it can work with most serial clustering methods as a pluggedin clustering subroutine, it balances the cost for disk accesses and network accesses, achieving a very good tradeoff between the two, it uses no user-defined parameters (thanks to our reasonable defaults), it matches the clustering quality of the serial algorithm, and it has near-linear scale-up; and finally, (3) We report experiments on real and synthetic data with billions of points, using up to 1,024 cores in parallel. To the best of our knowledge, our Yahoo! web is the largest real dataset ever reported in the database subspace clustering literature. Spanning 0.2 TB of multi-dimensional data, it took only 8 minutes to be clustered, using 128 cores.
\end{abstract}

\section{INTRODUCTION}

Given a very large dataset of moderate-to-high dimensional elements, how could one cluster them? Numerous successful, serial subspace clustering algorithms for data in five or more dimensions exist in literature. See [14] for a recent survey. However, the existing algorithms are impractical for datasets spanning Terabytes and Petabytes (e.g., Twitter crawl: $>12$ TB, Yahoo! operational data: 5 Petabytes [10]). In such cases, the data are already stored

\footnotetext{
* Work performed during Mr. Cordeiro's visit to CMU.
}

Permission to make digital or hard copies of all or part of this work for personal or classroom use is granted without fee provided that copies are not made or distributed for profit or commercial advantage and that copies bear this notice and the full citation on the first page. To copy otherwise, to republish, to post on servers or to redistribute to lists, requires prior specific permission and/or a fee.

Copyright 20XX ACM X-XXXXX-XX-X/XX/XX ...\$10.00. on multiple disks, since the largest modern disks are 1-2TB. Just to read a single Terabyte of data (at $5 \mathrm{~GB} / \mathrm{min}$ on a single modern eSATA disk) one takes more than 3 hours! Thus, parallelism is not another option - it is by far the best choice. Nevertheless, good, serial clustering algorithms and strategies are still extremely valuable, because we can (and should) use them as 'plug-ins' for parallel clustering. Naturally, the best algorithm is the one that combines (a) a fast, scalable serial algorithm and (b) makes it run efficiently in parallel. This is exactly what our proposed method does.

Examples of applications with Terabytes of data in five or more dimensions abound: weather monitoring systems and climate change models, where we want to record wind speed, temperature, rain, humidity, pollutants, etc; social networks like Facebook TM, with millions of nodes, and several attributes per node (gender, age, number of friends, etc); astrophysics data, such as the SDSS (Sloan Digital Sky Survey), with billions of galaxies and attributes like red-shift, diameter, spectrum, etc.

This paper focuses on the problem of finding subspace clusters in very large moderate-to-high dimensional data, that is, having typically more than 5 axes. Our method uses MapReduce, and can treat as plug-in most of the serial clustering methods. The major research challenges addressed are (a) how to minimize the I/O cost, taking into account the already existing data partition (e.g., on disks), and (b) how to minimize the network cost among processing nodes. Any of them may be a bottleneck. So, we propose the Best of both Worlds - BoW method, that automatically spots the bottleneck and picks a good strategy. Our main contributions are:

1. Algorithm design and analysis: we propose BoW, a novel, adaptive method to automatically pick the best of two strategies and proper parameters for it, one of the strategies uses a novel sampling-and-ignore idea to shrink the network traffic;

2. Effectiveness, scalability and generality: we show that BoW can work with most serial clustering methods as a plugged-in clustering subroutine, it balances the cost for disk accesses and network accesses, achieving a very good tradeoff between the two, it uses no user defined parameters (thanks to our defaults), and it maintains the serial clustering quality, with near-linear scale-up;

3. Experiments: we report experiments on real and synthetic data of billions of points, using up to 1,024 cores in parallel.

Figure 1 shows an example of $B o W$ 's results on real data. It plots the wall-clock-time versus the number of machines (MapReduce reducers), in log-log scales. The data consists of the top 10 eigenvectors of the adjacency matrix of the Twitter (http://twitter.com/) 


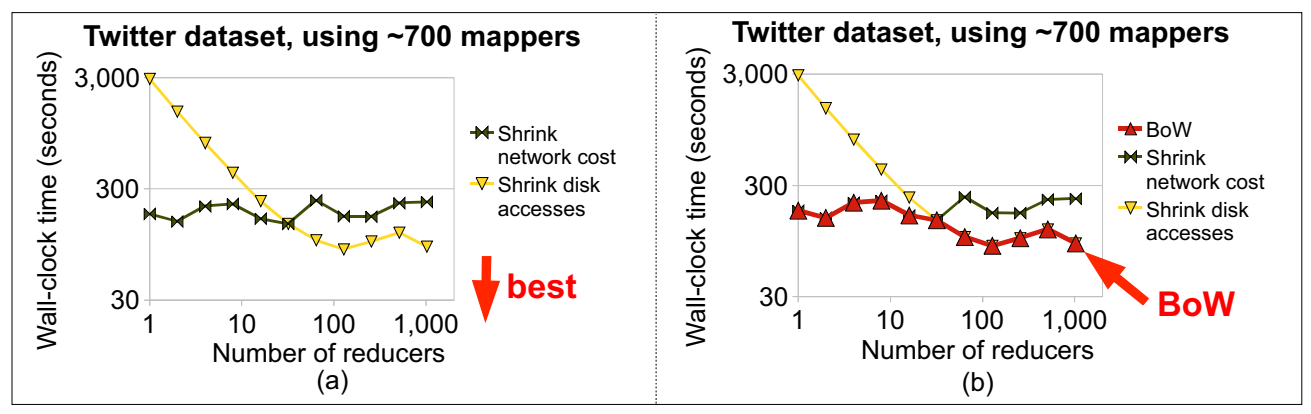

Figure 1: Results on real data from Twitter. Time vs. \# of machines (MapReduce reducers), in log-log scale. 700 MapReduce mappers used for all runs. Left: the upcoming ParC (yellow down-triangles) and SnI (dark-green butterflies) approaches. The latter uses our sampling-and-ignore idea; Right: the same, including $B o W$ (in red up-triangles). BoW uses cost-based optimization to pick the winning method and proper parameters for it, and thus practically over-writes the respective curve on the graph.

graph, which represents $\sim 62$ million users and their relationships. The eigenvectors span $\sim 14 G B$. The full details are in Section 5. Figure 1(a) shows the results for two of the best approaches we studied: the first, in yellow down-triangles, processes the whole dataset, while the second, in dark-green 'butterfly' glyphs, uses our proposed sampling-and-ignore idea. Notice that there is no universal winner, with a cross-over point at about 30 machines for this setting. Figure 1(b) shows exactly the same results, this time including the wall-clock time of our $B o W$, in red up-triangles. Notice that $B o W$ locks onto the best of the two alternatives, hence its name 'Best of both Worlds'. This is due to our upcoming cost-estimation formulas (Eq. (4) and (5)), which help BoW to pick the best alternative and set proper parameters for the chosen environment, while requiring nimble computational effort. Furthermore, notice that the two curves in Figure 1(a) intersect at a narrow angle, which means that the optimal curve has a smooth plateau, and thus the cost is rather robust wrt small variations of the environment parameters (like effective network bandwidth, disk transfer rate, file size, etc.).

We report experiments on real and synthetic, large datasets, including the Yahoo! web one. ${ }^{1}$ To the best of our knowledge, the Yahoo! web is the largest real dataset for which results have ever been reported in the database clustering literature for data in five or more axes. Although spanning $0.2 \mathrm{~TB}$ of multi-dimensional data, BoW took only 8 minutes to cluster it, using 128 cores. We also report experiments using 1,024 cores, the highest such number in the clustering literature for moderate-to-high dimensional data.

Notice that BoW is tailored to subspace clustering and can handle most serial algorithms as plug-ins, since the only required API is that the serial algorithm should return clusters of points in hyperrectangles, which we shall refer to as $\beta$-clusters. Subspace clustering methods spot clusters that exist only in subspaces of the original, $d$-dimensional space (i.e., spaces formed by subsets of the original axes or linear combinations thereof). Thus, the natural shape of the clusters in the original space facilitates their representation with hyper-rectangles, as the points of each cluster spread linearly through several irrelevant axes (original axes or linear combinations thereof) in the original space. For that reason, many serial, subspace clustering methods (e.g., CLIQUE [5], FPC/CFPC [19], $\mathrm{MrCC}[8], \mathrm{P} 3 \mathrm{C}$ [17] and STATPC [16]) return clusters in hyperrectangles, and adapting others to work with $\mathrm{BoW}$ tends to be facilitated by the clusters' natural shape. Nevertheless, besides focusing on subspace clustering and moderate-to-high dimensional data, $B o W$ also works with traditional clustering methods and low

\footnotetext{
${ }^{1}$ Provided by Yahoo! Research (www.yahoo.com).
}

dimensional data, if the plug-in returns clusters in hyper-rectangles.

The remaining of the paper comprises: related work (Section 2); proposed techniques (Sections 3 and 4); experiments (Section 5) and conclusions (Section 6). Table 1 lists the used symbols.

Table 1: Table of symbols.

\begin{tabular}{c|l}
\hline Symbols & Definitions \\
\hline${ }^{d} S$ & A $d$-dimensional dataset. \\
$d$ & Dimensionality of dataset ${ }^{d} S$. \\
$\eta$ & Cardinality of dataset ${ }^{d} S . \eta=\left|{ }^{d} S\right|$ \\
$k$ & Number of clusters in dataset ${ }^{d} S$. \\
$r$ & Number of reducers for parallel run. \\
$m$ & Number of mappers for parallel run. \\
$F_{s}$ & Database file size in bytes. \\
$D_{s}$ & Disk transfer rate in bytes/sec. \\
$N_{s}$ & Network transfer rate in bytes/sec. \\
$D_{r}$ & Dispersion ratio. \\
$R_{r}$ & Reduction ratio. \\
$S_{r}$ & Sampling ratio. \\
start_up_cost $(t)$ & Start-up cost for $t$ MapReduce tasks. \\
plug_in_cost $(s)$ & Serial clustering cost wrt the data size $s$. \\
\hline
\end{tabular}

\section{RELATED WORK}

\subsection{Subspace Clustering}

Clustering methods for data in five or more dimensions, known as subspace clustering methods, usually follow one of two approaches: density-based and $k$-means-based. A recent survey is found in [14]. Density-based methods assume that a cluster is a data space region in which the element distribution is dense. Each region may have an arbitrary shape and the elements inside it may be arbitrarily distributed. A cluster is separated from the others by regions of low density, whose points are considered as noise. The algorithms use own heuristics to identify dense and non-dense regions, usually relying on user-defined density thresholds. Examples of such algorithms are CLIQUE [5], COPAC [1], P3C [17], 4C [6], FIRES [13], FPC/CFPC [19], STATPC [16] and MrCC [8].

Methods like $k$-means start by picking $k$ space positions as clusters centroids, selected either by own heuristics or randomly. Clustering is achieved by an iterative process that assigns each point to its closest center, constantly improving the centers according to the points assigned to each cluster. The process stops when a quality criterion is satisfied or when a maximum number of iterations is achieved. Some of these methods are: PROCLUS [4], ORCLUS [3], PkM [2], CURLER [18] and LWC/CLWC [7]. 
Despite the several desirable properties found in existent methods, currently no subspace clustering algorithm is able to handle very large datasets in feasible time, and interesting datasets span way over the existing method's limits (e.g., Twitter crawl: $>12$ TB, Yahoo! operational data: 5 Petabytes [10]). For data that do not fit even on a single disk, parallelism is mandatory, and thus we must re-think, re-design and re-implement existing serial algorithms in order to allow for parallel processing.

\subsection{MapReduce}

MapReduce is a programming framework [9] to process largescale data in a massively parallel way. MapReduce has two major advantages: the programmer is oblivious of the details related to the data storage, distribution, replication, load balancing, etc.; and furthermore, it adopts the familiar concept of functional programming. The programmer must specify only two functions, a map and a reduce. The typical framework is as follows [15]: (a) the map stage passes over the input file and outputs (key, value) pairs; (b) the shuffling stage transfers the mappers' output to the reducers based on the key; (c) the reduce stage processes the received pairs and outputs the final result. Due to its scalability, simplicity and the low cost to build large clouds of computers, MapReduce is a very promising tool for large scale data analysis, something already reflected in academia (see [12] [11] for examples).

\section{PROPOSED MAIN IDEAS - REDUCING BOTTLENECKS}

The major research problems for clustering very large datasets with MapReduce are: (a) how to minimize the I/O cost, and (b) how to minimize the network cost among processing nodes. Should we split the data points at random, across machines? What should each node do, and how should we combine the results? Do we lose accuracy (if any), compared to a serial algorithm on a hugememory machine?

Our proposed method answers all these questions, by careful design and by adaptively trading-off disk delay and network delay.

In a nutshell, our proposed method $B o W$ is a hybrid between two methods that we propose next: the ParC method and the SnI. The former does data partitioning and merges the results; the latter does some sampling first, to reduce communication cost at the expense of higher I/O cost. Next, we describe each proposal in detail.

\subsection{Parallel Clustering - ParC}

The ParC algorithm has three steps: (1) appropriately partition the input data and assign each data partition to one machine, (2) each machine finds clusters in its assigned partition, named as $\beta$ clusters, and, (3) merge the $\beta$-clusters found to get the final clusters.

We considered three options for data partitioning, shortly described as follows due to space limitations: (a) random data partitioning: elements are assigned to machines at random, striving for load balance; (b) address-space data partitioning: eventually, nearby elements in the data space often end up in the same machine, trading-off load balance to achieve better merging of the $\beta$ clusters; and (c) arrival order or 'file-based' data partitioning: the first several elements in the collection go to one machine, the next batch goes to the second, and so on, achieving perfect load balance. The rationale is that it may also facilitate the merging of the $\beta$-clusters, because data elements that are stored consecutively on the disk, may also be nearby in address space, due to locality: For example, galaxy records from the Sloan Digital Sky Survey (SDSS) are scanned every night with smooth moves of the telescope, and thus galaxies close in (2-d) address space, often result in records that are stored in nearby locations on the disk.
Notice one observation: we performed an extensive experimental evaluation of the three partitioning approaches, which is omitted here due to space limitations. The file-based data partitioning was the fastest approach in our evaluation, still providing highly accurate results. Thus, the file-based approach is considered and used as the default strategy for the rest of this paper. Notice, however, that our methods work with the three partitioning approaches described, and, potentially, work with any user-defined partitioning strategy.

As described in Section 2.2, a MapReduce-based application has at least two modules: the map and the reduce. Our ParC method partitions the data through MapReduce mappers and does the clustering in MapReduce reducers. The final merging is performed serially, since it only processes the clusters descriptions, which consist of a tiny amount of data and processing. Figure $2 \mathrm{a}$ ( $2 \mathrm{~b}$ will be explained latter) illustrates the process. It starts in phase $\mathbf{P 1}$ with $m$ mappers reading the data in parallel from the MapReduce distributed file system. In this phase, each mapper receives a data element at a time, computes its key, according to the data partition strategy used, and outputs a pair $\langle k e y$, point $\rangle$. All elements with the same key are forwarded in phase $\mathbf{P 2}$ to be processed together, by the same reducer, and the elements with distinct keys are processed apart, by distinct reducers.

In phase P3, each reducer receives its assigned set of elements and normalizes them to a unitary hyper-cube. Each reducer then applies the plugged-in serial clustering algorithm over the normalized elements, aiming to spot $\beta$-clusters. For each $\beta$-cluster found, the reducer outputs, in phase $\mathbf{P 4}$, a pair $\langle k e y$,cluster_description $\rangle$. The key concatenates the reducer identification and a cluster identification. The reducer identification is the input key. The cluster identification is a sequential number according to the order in which the $\beta$-cluster was found in the corresponding reducer. A $\beta$-cluster description consists of the unnormalized minimum/maximum bounds of the cluster in each dimension, defining a hyper-rectangle in the data space. Notice that this is a tiny amount of data, amounting to two float values per axis, per $\beta$-cluster.

The final phase $\mathbf{P 5}$ is performed serially, as it processes only the tiny amount of data ( $\beta$-clusters' bounds) received from phase $\mathbf{P 4}$, and not the data elements themselves. Phase P5 merges all $\beta$-clusters pairs that overlap in the data space. Checking if two $\beta$ clusters overlap refers to checking if two hyper-rectangles overlap in a $d$-dimensional space.

\subsection{Sample-and-Ignore - SnI}

The first algorithm, ParC, reads the dataset once, aimed at minimizing disk accesses, which is the most common strategy used by serial algorithms to shrink computational costs. However, this strategy does not address the issue of minimizing the network traffic: in the shuffle phase of the ParC algorithm (phase $\mathbf{P 2}$ of Figure 2a), almost all of the records have to be shipped over the network, to the appropriate reducer. How can we reduce this network traffic?

Our main idea is to exploit the skewed distribution of cluster sizes that typically appears in real datasets: Most of the elements usually belong to a few large clusters, and these are exactly the elements that we try to avoid processing. Thus, we propose $S n I$, a parallel clustering algorithm that consists of: (a) a novel sampleand-ignore preprocessing step; and (b) the ParC algorithm from Section 3.1. The sample-and-ignore step works on a small dataset sample, spots the major clusters and ignores their members in the follow-up steps. It significantly reduces the amount of data moved in the shuffling phases of $S n I$, with consequent savings for the network traffic, as well as the $\mathrm{I} / \mathrm{O}$ cost for the intermediate results and processing cost for the receiving reduce tasks. Notice one point: the proposed sample-and-ignore idea is an alternative general strategy 
(a) Parallel processing for ParC

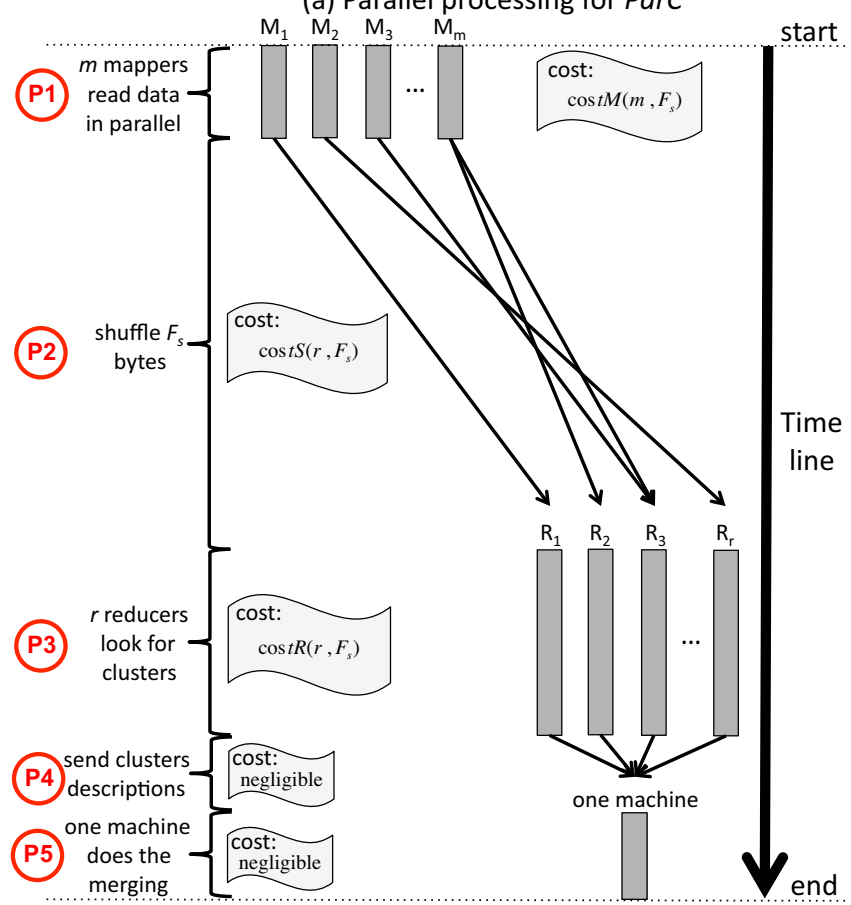

(b) Parallel processing for $\mathrm{SnI}$

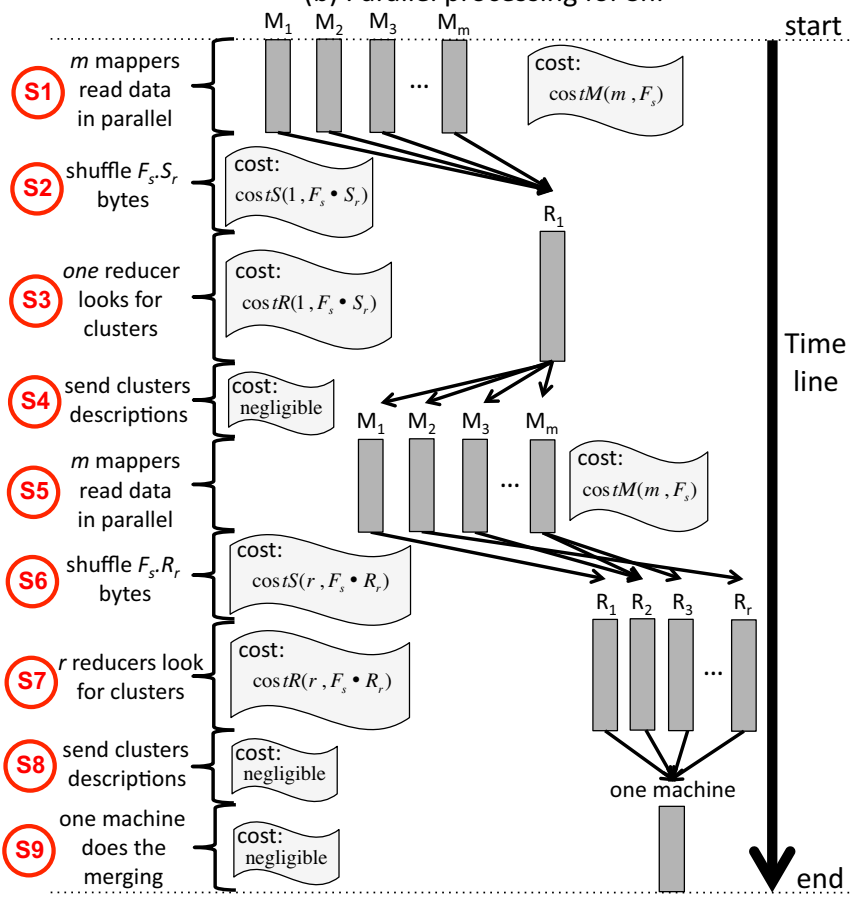

Figure 2: Which one is best? Parallel run overview for ParC (left) and SnI (right - with sampling). ParC executes the map (PI), shuffle $(P 2)$ and reduce $(P 3)$ phases once, on the full dataset. $S n I$ uses sampling (phases $S 1-S 4$ ) to get rough cluster estimates and then uses phases $S 5-S 9$ to cluster the remaining points (see section 3.2 for details). Their clustering qualities are similar (see Section 5). The winning approach depends on the environment; $B o W$ uses cost-based optimization to automatically pick the best.
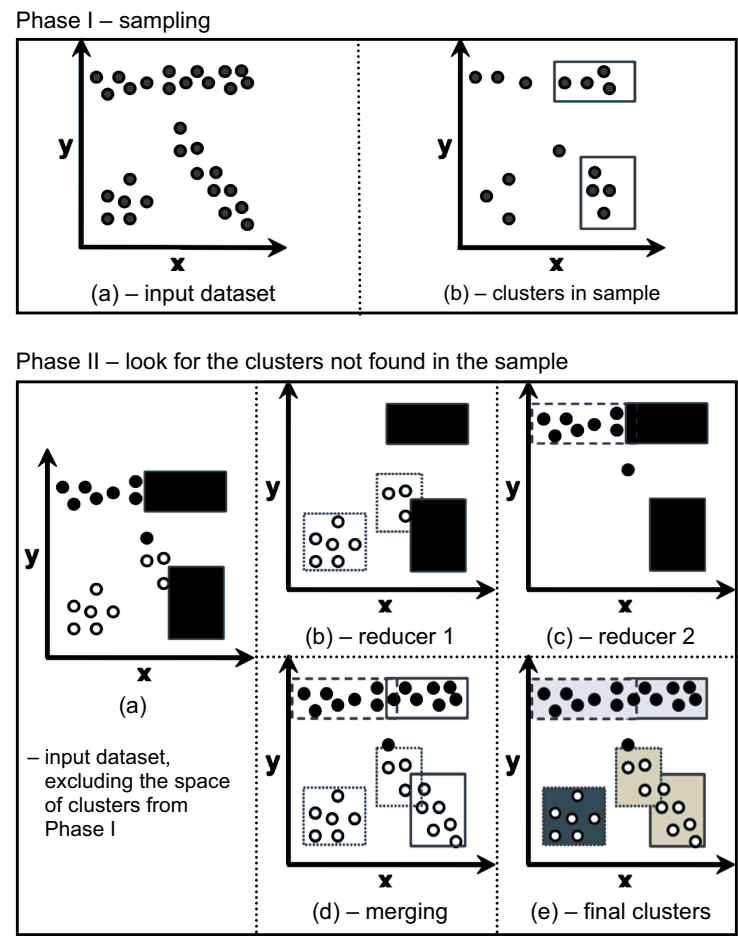

Figure 3: Multi-phase Sample-and-Ignore (SnI) Method. Phase-I finds clusters on a sample of the input data. Phase-II ignores elements that fall within any previously found cluster and finds clusters using the remaining elements only. that can improve many clustering methods, and not only ParC.

The $S n I$ method is defined in Algorithm 1 and the process is illustrated in Figure 2b. At a high-level, in Phase I (steps S1-S4 in the figure, and lines 1-3 in the algorithm) the method samples the input data and builds an initial set of clusters. In the second phase (steps S5-S9 in the figure, and lines 4-8 in the algorithm), the input data is filtered, so that we only include unclassified elements, that is, those that do not belong to any of the clusters found in Phase I. These unclassified elements are then clustered using ParC.

Figure 3 illustrates the $S n I$ approach over a toy dataset, assuming that we have $r=2$ reducers available for parallel processing. The top part of the figure shows Phase-I. First, in Phase-I (a) the input dataset is read in parallel by $m$ map tasks, each mapper passes the input elements to the same reducer with some probability, for example, 0.5 for the case shown in the figure. A single reducer builds clusters using the sample elements in Phase-I (b). In this case two clusters were found and are denoted by the gray boxes around the elements. The summary descriptors of the clusters found in PhaseI, i.e., the minimum/maximum limits of the clusters wrt each dimension, are passed to Phase-II. In Phase-II (a), $m$ mappers perform a second pass over the data, this time filtering out elements that fall in the clusters found in Phase-I, which are denoted by the black boxes. The elements that do not fall into clusters are passed to the two reducers available, as shown in Phase-II (b) and (c), in which we assume that the used partitioning strategy divided the elements into 'black points' and 'white points'. Each reducer finds new clusters, denoted by the points surrounded by dotted boxes. In Phase-II (d), the clusters found by the reducers are merged with the clusters from the sampling phase using the same merging strategies used in $\operatorname{ParC}$. The global set of clusters, with three clusters represented in Phase-II (e) by distinct gray levels, is the final output. 
The main benefit of the $S n I$ approach is realized in the shuffle/reduce stages. In Phases $\mathbf{S 2}$ and $\mathbf{S 3}$ of Figure 2b, only a small sample is shuffled and processed by a receiving reducer. In Phases S6 and S7 of Figure 2b, only the non-ignored elements may need to be shuffled through the network to other machines and processed. This means that most elements belonging to the major clusters spotted in the sample are ignored, never being shuffled through the network nor processed by a reducer. Compared to the $\operatorname{ParC}$ algorithm, $\mathrm{SnI}$ significantly minimizes the network cost and the reducers processing, at the cost of reading the whole dataset twice. In other words, ParC does a single pass over the data, but almost all of the records have to be shipped over the network (phase P2 of Figure $2 \mathrm{a})$, to the appropriate reducer. On the other hand, SnI minimizes the shuffle/reduce cost, at the expense of reading the data one extra time. What approach is the best? The answer is given in Section 4.

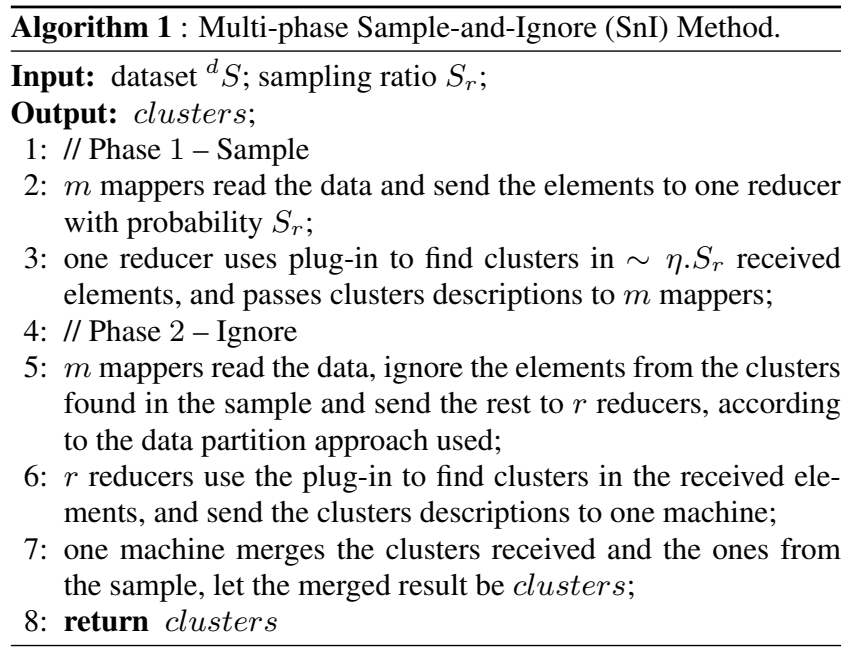

\section{PROPOSED COST-BASED OPTIMIZATION}

We propose an adaptive, hybrid method named BoW (Best of both Worlds) that exploits the advantages of the previously described approaches, ParC and SnI, taking the best of them. There is no universal winner, since it depends on the environment and on the data characteristics. See Figure 1 and Section 5 for a complete explanation. Therefore, the main question here is: When should our sampling-and-ignore idea be used and when should it be avoided? $\operatorname{ParC}$ runs the map, shuffle and reduce phases only once on the whole dataset. SnI reduces the amount of data to be shipped to and processed by the reducers, at the cost of a second pass on the input data (in the map phase). We propose a cost-based optimization that uses analytics models to estimate the running time of each clustering strategy. BoW picks the one with the lowest estimated cost.

The environmental parameters required by $B o W$ are presented in Table 2. They describe the hardware characteristics (i.e., the specs of the available MapReduce cluster), the total amount of data to be processed, and the cost estimate for the plugged-in serial clustering method. Setting the value for $F_{s}$ is straightforward. $D_{s}$, $N_{s}$ and start_up_cost $(t)$ are inferred by analyzing the cloud of computers' logs, while plug_in_cost $(s)$ is defined based on the plugged-in method's original time complexity analysis and/or experiments, or measured by the user in a simple experiment. Notice: each machine in the cloud may run many MapReduce tasks (mappers and/or reducers) in parallel, sharing the machine's disks and network connection. So, $N_{s}$ and $D_{s}$ are expected to be smaller than the effective network bandwidth and disk transfer rate respectively.

Table 2: Environmental parameters

\begin{tabular}{|c|c|c|}
\hline Parameter & Meaning & Explanation \\
\hline$\overline{F s}$ & $\begin{array}{l}\text { data file size } \\
\text { (bytes) }\end{array}$ & $\begin{array}{l}\text { Size of the dataset } \\
\text { to be clustered. }\end{array}$ \\
\hline$D_{s}$ & $\begin{array}{l}\text { disk speed } \\
\text { (bytes/sec.) }\end{array}$ & $\begin{array}{l}\text { Average bytes/sec. that } \\
\text { a MapReduce task } \\
\text { (mapper or reducer) can } \\
\text { read from local disks. }\end{array}$ \\
\hline$N_{s}$ & $\begin{array}{l}\text { network speed } \\
\text { (bytes/sec.) }\end{array}$ & $\begin{array}{l}\text { Average bytes/sec. that } \\
\text { a MapReduce task } \\
\text { (mapper or reducer) } \\
\text { can read from other } \\
\text { computers in the cloud. }\end{array}$ \\
\hline start_up_cost $(t)$ & $\begin{array}{l}\text { start-up cost } \\
\text { (seconds) }\end{array}$ & $\begin{array}{c}\text { Average time to start-up } \\
t \text { MapReduce tasks } \\
\text { (mappers or reducers). }\end{array}$ \\
\hline plug_in_cost $(s)$ & $\begin{array}{l}\text { plug-in cost } \\
\text { (seconds) }\end{array}$ & $\begin{array}{l}\text { Average time to run } \\
\text { the plugged-in serial } \\
\text { method over } s \text { data } \\
\text { bytes on a standard } \\
\text { computer in the cloud. }\end{array}$ \\
\hline
\end{tabular}

Two other parameters are used, shown in Table 3. We provide reasonable default values for them based on empirical evaluation. Notice one important observation: As is the common knowledge in database query optimization, at the cross-over point of two strategies, the wall-clock-time performances usually create flat plateaus, being not much sensitive to parameter variations. This occurs in our setting, and the results in Figures 1a, 7a and 7d exemplify it (notice the log-log scale). Thus, tuning exact values to our parameters barely affects $B o W$ 's results and the suggested values are expected to work well in most cases.

Table 3: Other parameters

\begin{tabular}{|c|c|c|c|}
\hline Param. & Meaning & Explanation & $\begin{array}{c}\text { Our } \\
\text { defaults }\end{array}$ \\
\hline \hline$D_{r}$ & $\begin{array}{c}\text { dispersion } \\
\text { ratio }\end{array}$ & $\begin{array}{c}\text { Ratio of data transferred in } \\
\text { the shuffling through the } \\
\text { network relative to the total } \\
\text { amount of data involved. }\end{array}$ & 0.5 \\
\hline$R_{r}$ & $\begin{array}{c}\text { reduction } \\
\text { ratio }\end{array}$ & $\begin{array}{c}\text { Ratio of data that does not } \\
\text { belong to the major clus- } \\
\text { ters found in the sampling } \\
\text { phase of } S n I \text { relative to } \\
\text { the full data size } F_{s} .\end{array}$ & 0.1 \\
\hline
\end{tabular}

The following lemmas and proofs define the equations of our cost-based optimization. First, we give the expected costs for the map, shuffle and reduce phases wrt the number of mappers and/or reducers available and to the data size involved. Then, we infer the costs for: ParC, that minimizes disk accesses, and; SnI, that aims at shrinking the network cost. For clarity, consider again Figure 2 that provides a graphical overview of the parallel execution of both methods, as well as their expected cost equations.

LEMMA 1. Map Cost - the expected cost for the map phase of the parallel clustering approaches is a function of the number of mappers $m$ used and the involved data size s, given by:

$$
\operatorname{cost} M(m, s)=\text { start_up_cost }(m)+\frac{s}{m} \cdot \frac{1}{D_{s}}
$$


PROOF. In the map phase, $m$ mappers are started-up at the cost of start_up_cost $(m)$. The majority of the extra time spent is related to reading the input data from disk. $s$ bytes of data will be read in parallel by $m$ mappers, which are able to read $D_{s}$ bytes per second each. Thus, the total reading time is given by: $\frac{s}{m} \cdot \frac{1}{D_{s}}$.

LEMMA 2. Shuffle Cost - the expected shuffle cost is a function of the number of reducers $r$ to receive the data and the amount of data to be shuffled s, which is given by:

$$
\operatorname{cost} S(r, s)=\frac{s \cdot D_{r}}{r} \cdot \frac{1}{N_{s}}
$$

PROOF. The majority of the shuffling cost is related to shipping the data between distinct machines through the network. Whenever possible, MapReduce minimizes this cost by assigning reduce tasks to the machines that already have the required data in local disks. $D_{r}$ is the ratio of data actually shipped between distinct machines relative to the total amount of data processed. Thus, the total amount of data to be shipped is $s . D_{r}$ bytes. The data will be received in parallel by $r$ reducers, each one receiving in average $N_{s}$ bytes per second. Thus, the total cost is given by: $\frac{s \cdot D_{r}}{r} \cdot \frac{1}{N_{s}}$.

Lemma 3. Reduce Cost - the expected cost for the reduce phase is a function of the number of reducers $r$ used for parallel processing and the size s of the data involved, which is given by:

$$
\begin{aligned}
\operatorname{cost} R(r, s)= & \operatorname{start\_ up\_ cost}(r)+\frac{s}{r} \cdot \frac{1}{D_{s}}+ \\
& p l u g \_i n \_c o s t\left(\frac{s}{r}\right)
\end{aligned}
$$

PRoOF. In the reduce phase, $r$ reducers are started-up at cost start_up_cost $(r)$. After the start-up process, the reducers will read from disk $s$ bytes in parallel at the individual cost of $D_{s}$ bytes per second. Thus, the total reading time is $\frac{s}{r} \cdot \frac{1}{D_{s}}$. Finally, the plugged-in serial clustering method will be executed in parallel over partitions of the data, whose average sizes are $\frac{s}{r}$. Therefore, the approximate clustering cost is plug_in_cost $\left(\frac{s}{r}\right)$.

Lemma 4. ParC Cost-the expected cost for ParC is:

$$
\operatorname{cost} C=\operatorname{cost} M\left(m, F_{s}\right)+\operatorname{cost} S\left(r, F_{s}\right)+\operatorname{cost} R\left(r, F_{s}\right)
$$

PRoOF. The parallel processing for $\operatorname{ParC}$ is: (i) $m$ mappers process $F_{s}$ bytes of data in the map phase; (ii) $F_{s}$ bytes of data are shuffled to $r$ reducers in the shuffling phase; (iii) $F_{s}$ bytes of data are analyzed in the reduce phase by $r$ reducers, and; (iv) a single machine merges all the $\beta$-clusters found. The last step has negligible cost, as it performs simple computations over data amounting to two float values per $\beta$-cluster, per dimension. Thus, summing the costs of the three initial phases leads to the expected cost.

LEMMA 5. SnI Cost - the expected cost for $\mathrm{SnI}$ is:

$$
\begin{aligned}
\operatorname{cost} C s= & 2 \cdot \operatorname{cost} M\left(m, F_{s}\right)+ \\
& \operatorname{cost} S\left(1, F_{s} \cdot S_{r}\right)+\operatorname{cost} R\left(1, F_{s} \cdot S_{r}\right)+ \\
& \operatorname{cost} S\left(r, F_{s} \cdot R_{r}\right)+\operatorname{cost} R\left(r, F_{s} \cdot R_{r}\right)
\end{aligned}
$$

Proof. SnI runs two complete map, shuffle and reduce phases. In both map phases, the full dataset is processed by $m$ mappers, at combined cost: $2 \cdot \operatorname{cost} M\left(m, F_{s}\right)$. In the first shuffle phase,

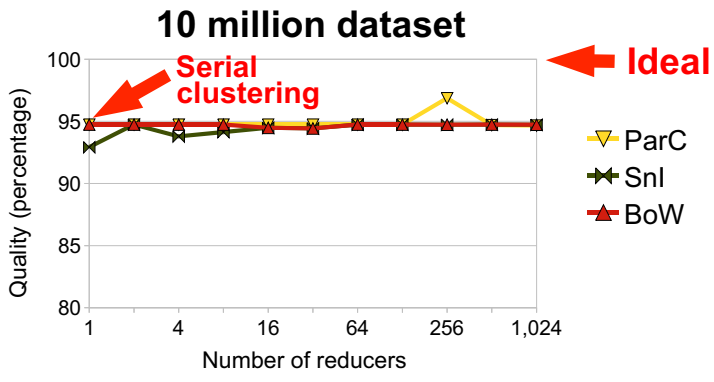

Figure 4: All our variants give top quality results. 10 million dataset; quality vs. number $r$ of reducers, for $\operatorname{ParC}, \mathrm{SnI}$ and $B o W$. All methods match the quality of the serial clustering method (top left), for all values of $r$, like 1,024 .

a data sample of size $F_{s} . S_{r}$ bytes is shuffled to a single reducer, at cost $\operatorname{cost} S\left(1, F_{s} . S_{r}\right)$. The reduce cost to process this sample is: $\operatorname{cost} R\left(1, F_{s} \cdot S_{r}\right) . R_{r}$ is the ratio of data that does not belong to the major clusters, the ones found in the sampling phase, relative to $F_{s}$. That is, $F_{s} .\left(1-R_{r}\right)$ bytes are ignored in the Second Phase of SnI, while $F_{s} . R_{r}$ bytes of data are not ignored, being processed after clustering the sample. Also, both second shuffle and reduce phases involve $r$ reducers. Thus, their combined costs are: $\operatorname{cost} S\left(r, F_{s} \cdot R_{r}\right)+\operatorname{cost} R\left(r, F_{s} \cdot R_{r}\right)$. The costs for shipping and processing $\beta$-clusters descriptions is negligible, since the involved amount of data and processing is extremely small.

Notice one observation: when our algorithms are executed, the number of distinct key values to be sorted by the MapReduce framework is tiny; it is always the number $r$ of reducers used only. Each reducer handles a single key, so it does not need to do sorting. Thus, the sorting cost is negligible for our approaches. The I/O and network costs are the real bottlenecks. The wall-clock time results in all of our experiments (see Section 5) confirm this assertion.

Algorithm 2 describes the main steps of BoW. In summary, ParC executes the map, shuffle and reduce phases once, involving the full dataset. SnI runs these phases twice, but involving less data. What is the fastest approach? It depends on your environment. $B o W$ takes the environment description as input and uses cost-based optimization to automatically choose the fastest, prior to the real execution. Provided that the clustering accuracies are similar for both approaches (see Section 5 for a complete explanation), BoW actually picks the 'Best of both Worlds'.

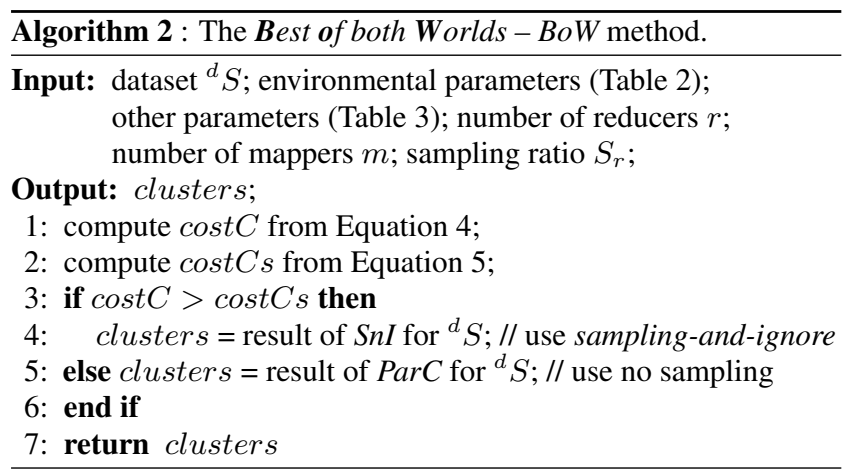

\section{EXPERIMENTAL RESULTS}

In this section, we describe the experiments performed. We aimed at answering the following questions: 


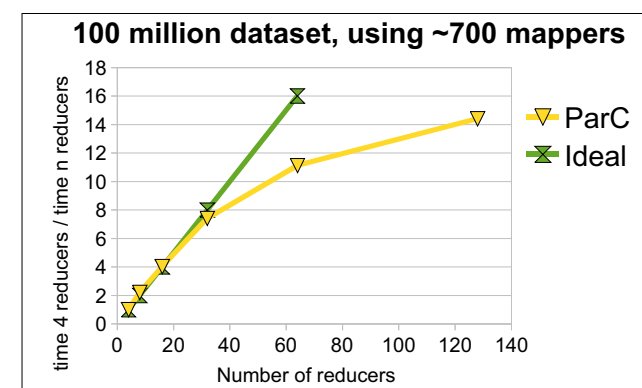

(a)

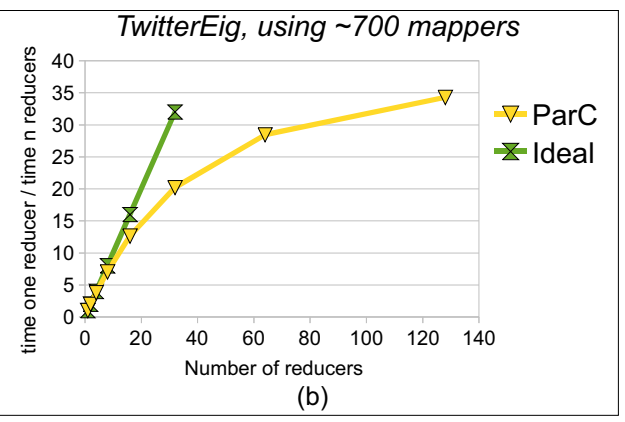

(b)

Figure 5: Near-linear scale-up wrt the number of reducers. Expected behavior: our method starts with near-linear scale-up, and then flattens. 100 million dataset (left); TwitterEig (right). X-axes: \# of reducers, Y-axes: the relative performance with $r$ reducers compared to that with 1 reducer (right) or 4 reducers (left). $r=1$ in the left case needs prohibitively long time. $\sim 700$ mappers used.

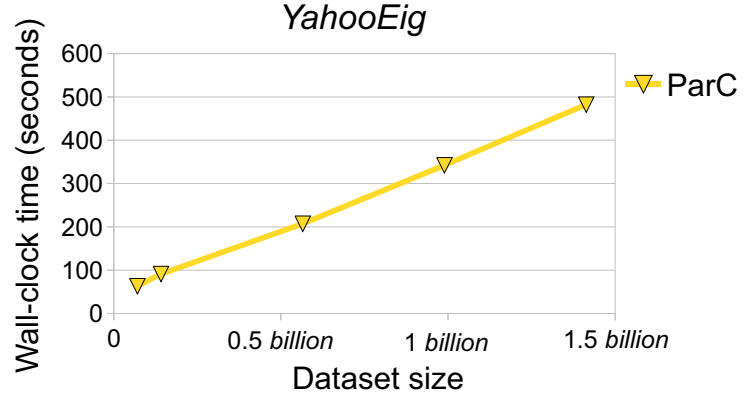

Figure 6: Linear scale-up on db size: wall-clock time vs. data size. Random samples from Yah००E ig, up to 1.4 billion points. Fixed numbers of reducers (128) and mappers $(\sim 700)$.

Q1 How much (if at all) parallelism affects the cluster's quality? Q2 How does our method scale-up?

Q3 How accurate are our cost-based optimization equations?

All experiments were done using the Hadoop ${ }^{2}$ implementation for the MapReduce framework, on two Hadoop clusters: M45 by Yahoo! and DISC/Cloud by Parallel Data Lab in CMU. M45 is one of the top 50 supercomputers in the world totaling 400 machines (3, 200 cores), $1.5 \mathrm{~PB}$ of storage and $3.5 \mathrm{~TB}$ of main memory. DISC/Cloud has 512 cores, distributed in 64 machines, 1TB of RAM and 256 TB of raw disk storage. We used the real and synthetic datasets described in Table 4, which are detailed as follows.

- YahooEig: The top 6 eigenvectors from the adjacency matrix of one of the largest web graphs. The web graph was crawled by Yahoo! ${ }^{3}$ in 2002 and contains 1.4 billion nodes and 6.6 billion edges. The eigenvectors amount to $0.2 \mathrm{~TB}$.

- TwitterEig: The top 10 eigenvectors from the adjacency matrix of the Twitter ${ }^{4}$ graph, that represents 62 million users and their relationships. Eigenvectors amount to 0.014 TB.

- Synthetic: A group of datasets with sizes varying from 100 thousand up to 100 million 15 -dimensional points, containing 10 clusters each. We created clusters following standard procedures used by most of the clustering algorithms cited in Section 2, including the plugged-in serial method used in our experiments. All clusters follow normal distributions with random means and random standard deviations in

\footnotetext{
${ }^{2}$ www.hadoop.com

${ }^{3}$ www.yahoo.com

${ }^{4}$ http://twitter.com/
}

at least $50 \%$ of the axes, spreading through at most $15 \%$ of these axes domains. In other axes, all clusters have uniform distribution, spreading through the whole axes domains.

Table 4: Summary of datasets. TB: TeraBytes

\begin{tabular}{|c|c|c|c|}
\hline Dataset & \# of Points & \# of Axes & File Size \\
\hline \hline YahooEig & 1.4 billion & 6 & $0.2 \mathrm{~TB}$ \\
\hline TwitterEig & 62 million & 10 & $0.014 \mathrm{~TB}$ \\
\hline Synthetic & up to 100 million & 15 & up to $0.014 \mathrm{~TB}$ \\
\hline
\end{tabular}

As our real world datasets have 6 and 10 axes, we chose the $\mathrm{MrCC}$ algorithm as the serial clustering method for the plug-in in all experiments. $\mathrm{MrCC}$ is one state-of-the-art clustering method for medium-dimensionality data. Its original code was used.

Notice one observation: to evaluate how much (if at all) parallelism affects the serial clustering quality, the ideal strategy is to use as ground truth the clustering results obtained by running the plugged-in algorithm serially on any dataset, synthetic or real, and to compare these results to the ones obtained with parallel processing. But, for most of our large datasets, to run a serial algorithm (MrCC or, potentially, any other clustering method for moderateto-high dimensionality data) is an impractical task - it would require impractical amounts of main memory and/or take a very long time. Thus, in practice, the Synthetic datasets are the only ones from which we have clustering ground truth, and they were used to evaluate the quality of all tested techniques in all experiments.

For a fair comparison with the plugged-in serial algorithm, the quality is computed following the same procedure used in its original publication. That is, the quality is computed by comparing the results provided by a technique to the ground truth, based on the averaged precision and recall of all clusters.

The file-based data partitioning strategy used may provide distinct quality results wrt the order in which the input data is physically stored. Obviously, the best results appear when the data is totally ordered, i.e., the elements of each cluster are sequentially stored in the data file. On the other hand, when the elements are randomly positioned in the file, the qualities are similar to the ones obtained when using the random data partitioning. For a fair analysis, we built each dataset from the Synthet ic group considering an average case, i.e., $50 \%$ of the elements from the totally ordered case were randomly repositioned throughout the data file.

The following values were used for the environmental parameters: $F_{s}$ : the data file size; $D_{s}: 40 \mathrm{MB} / \mathrm{sec} ; N_{s}: 20 \mathrm{MB} / \mathrm{sec}$; start_up_cost $(t): 0.1 t ; p l u g \_i n \_c o s t(s): 1.4 E^{-7} s$. They were measured by experiments on the M45 machines and on the serial 


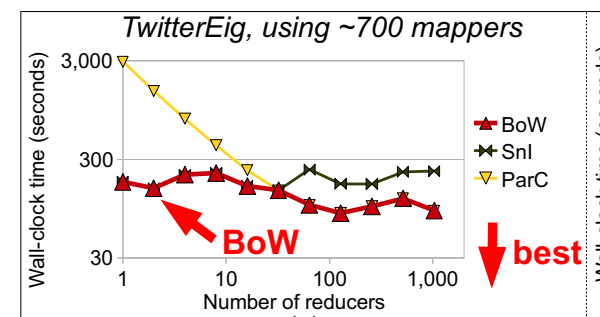

(a)

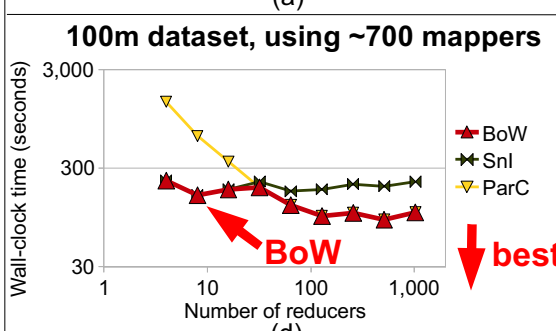

(d)

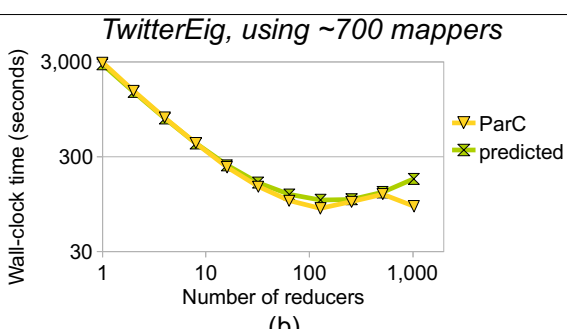

(b)

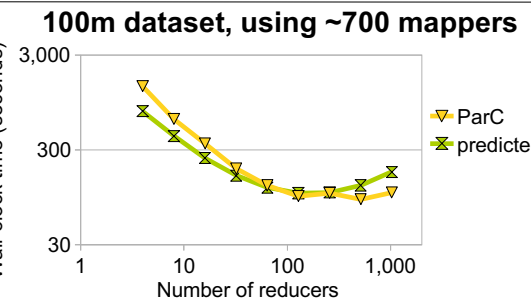

(e)

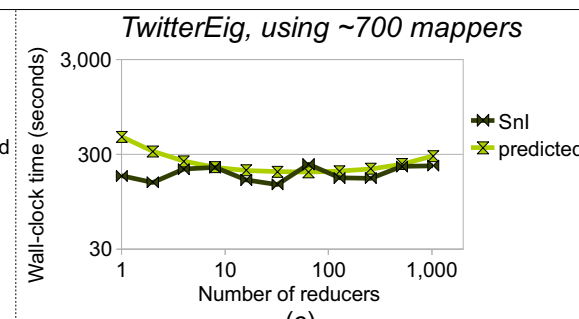

(c)

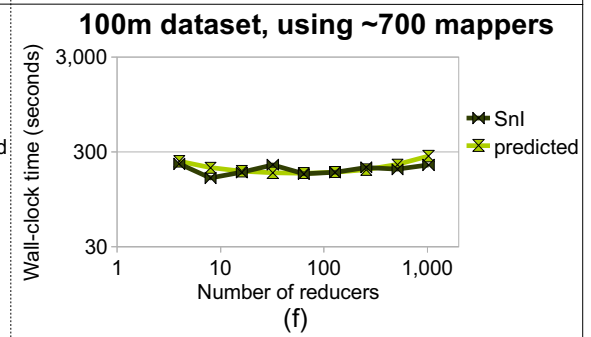

Figure 7: Top: TwitterEig; Bottom: Synthetic, 100 million. Time vs. \# of reducers, in log-log scale. $\sim 700$ mappers always. Left column: BoW's ability to pick the winner, among ParC (yellow down-triangles) and $S n I$ (dark-green butterflies). BoW (red uptriangles) gets the best of both, always picks the winning strategy, and thus practically over-writes the winner's curve. Middle/right columns: accuracy of our Equations 4 and 5; In all cases, the light-green hour-glass shapes stand for our formulas; Notice how close they are to the actual measurements (ParC in yellow triangles and $\mathrm{SnI}$ in dark-green butterflies).

plug-in to represent the environment employed. Details on this procedure are not shown due to space limitation. All experiments involving $B o W$ used these parameters and the M45 machines.

The results on quality and wall-clock time shown for all experiments are averages of 10 distinct runs. All experiments used a sample size of $\sim 1$ million, i.e., $S_{r}=\frac{1 \text { million }}{\eta}$, and, in all cases, the number of mappers $m$ used was automatically chosen by Hadoop.

\subsection{Quality of results}

This section intends to answer question $Q 1$ : How much (if at all) does the parallelism affect the clustering quality? Figure 4 shows the quality results obtained by $\operatorname{ParC}, \mathrm{SnI}$ and $\mathrm{BoW}$ over our Synthetic dataset with 10 million elements. All tested methods presented top quality, even for large numbers of reducers, like 1,024 . Notice, that the serial execution quality of the plugged-in clustering method is the one obtained when using a single reducer ( $r=1$, extreme left in the plot). Similar results were observed with the other Synthetic datasets, not shown for brevity.

An interesting observation is that the quality may decrease for small datasets, when using a large number of reducers. The obvious reason is that, in those cases, we are partitioning a small amount of data through a large number of reducers, which actually receive too little data, not enough to represent the main data patterns. This fact was confirmed in all our experiments, and they lead us to recommend at least $\sim 150 k$ points per reducer in average, i.e., $r \leq \frac{\eta}{150 k}$.

Thus, based on our experiments, the answer to question $Q 1$ is: as long as you have enough data, parallelism barely affects the accuracy, even for large numbers of reducers, like 1, 024. BoW found top quality clusters in little time from all our very large datasets.

\subsection{Scale-up results}

This section intends to answer question $Q 2$ : How does our method scale-up? Scale-up results with different numbers of reducers are in Figure 5. Here we used the TwitterEig eigenvectors and the Synthetic dataset with 100 million points. The plots show $\mathrm{X}$-axes as the number of reducers $r$, and the Y-axes as the relative performance with $n$ reducers compared to that with 1 reducer
(TwitterEig) or 4 reducers (Synthetic). A fixed number of mappers $m=\sim 700$ was used. The results shown are the average of 10 distinct runs. We picked 4 reducers for our Synthet ic dataset, as the running time for one reducer was impractical. Notice that our method achieves near-linear scale-up.

The scale-up results with different data sizes are in Figure 6. The YahooEig dataset is used. Random samples of the data with increasing sizes, up to the full dataset (1.4 billion elements) were generated to perform this experiment. We plot wall clock time vs. data size. The wall-clock time shown is the average time of 10 distinct runs. Fixed numbers of reducers and mappers $(r=128$ and $m=\sim 700$ ) were used. As shown, our method has desired scalability, scaling-up linearly with the data size.

It took only $\sim 8$ minutes to cluster the full dataset, which amounts to $0.2 T B$ ! Let's provide some context to this result by characterizing the time taken at different stages in the process: (a) the mappers took 47 seconds to read the data from disks; (b) 65 seconds were taken to shuffle the data; and (c) the reduce stage took 330 seconds. To estimate the time taken by the serial method in item (c), we clustered a random sample of the YahooEig dataset, of size $\frac{F_{s}}{r}=\frac{0.2 T B}{128}$, by running the plug-in on a single machine (one core), similar to the ones of the used cloud of computes. The serial clustering time was 192 seconds. This indicates that the plugin took $\sim 43 \%$ of the total time, being the main bottleneck.

Similar scale-up results were obtained for all other datasets, not shown here due to the space limitation.

\subsection{Accuracy of our cost equations}

Here we refer to question Q3, by checking BoW's ability to pick the correct alternative and the accuracy of our cost formulas, (Eq. (4) and (5)) from Section 4. The results for the TwitterEig and Synthetic (with 100 million points) datasets are shown in the top and bottom lines of Figure 7, respectively. The six plots give the wall-clock time (average of 10 runs) versus the number of reducers $r$, in log-log scales. The left column ((a) and (d)) shows that $B o W$, in red up-triangles, consistently picks the winning strat- 
egy among the two alternatives: ParC (yellow down-triangles) and SnI (dark-green butterflies), that uses our sample-and-ignore idea. For both datasets, $B o W$ gives results so close to the winner, that its curve practically overwrites the winner's curve; the only overhead of $B o W$ is the CPU time required to run the cost equations, which is negligible. The next two columns of Figure 7 illustrate the accuracy of our cost formulas. Light-green hour-glasses refer to our theoretical prediction; yellow triangles stand for ParC (middle column), and dark-green butterflies stand for $S n I$ (right column). Notice: the theory and the measurements usually agree very well. All other datasets gave similar results, omitted for brevity.

\section{CONCLUSIONS}

Given a very large moderate-to-high dimensionality dataset, how could one cluster its points? For data that don't fit even on a single disk, parallelism is mandatory. The bottlenecks are then: $\mathrm{I} / \mathrm{O}$ cost and network cost. Our main contributions are:

1. Algorithm design and analysis: We proposed BoW and carefully derived its cost functions that allow the automatic, dynamic trade-off between disk delay and network delay;

2. Effectiveness, scalability and generality: We showed that BoW has many desirable features: it can work with most serial methods as a plugged-in clustering subroutine (the only API: clusters described by hyper-rectangles), it balances the cost for disk accesses and network accesses, achieving a very good tradeoff between the two, it uses no user defined parameters (thanks to our defaults) and it matches the serial algorithm's clustering accuracy with near-linear scale-up;

3. Experiments: We report experiments on real and synthetic data of billions of points, using up to 1,024 cores in parallel.

To the best of our knowledge, the Yahoo! web is the largest real dataset ever reported in the database subspace clustering literature. $B o W$ clustered its $0.2 T B$ in only 8 minutes, with 128 cores!

Finally, notice that $B o W$ is a hard-clustering method and, as well as any other method of this type, it may not be the best solution for data with many overlapping clusters. So, we report an idea for future work: to extend $B o W$ 's merging stage to return soft-clustering results, allowing any data point to belong to two or more clusters. In our opinion, soft-clustering is a promising idea, but there are several issues involved, which are out of the scope of this paper.

\section{ACKNOWLEDGMENTS}

This material is based upon work supported by FAPESP (São Paulo State Research Foundation), CAPES (Brazilian Coordination for Improvement of Higher Level Personnel), CNPq (Brazilian National Council for Supporting Research), Microsoft Research, the National Science Foundation (NSF), under award CCF-1019104, the Qatar National Fund, under award NPRP 09-1114-1-172 in the QCloud project, the Gordon and Betty Moore Foundation, in the eScience project, the Defense Threat Reduction Agency, accomplished under contract No. HDTRA1-10-1-0120, and the Army Research Laboratory, accomplished under Cooperative Agreement Number W911NF-09-2-0053. The views and conclusions contained in this document are those of the authors and should not be interpreted as representing the official policies, either expressed or implied, of the Army Research Laboratory, the U.S. Government, the National Science Foundation, or other funding parties. The U.S. Government is authorized to reproduce and distribute reprints for Government purposes notwithstanding any copyright notation here on. We also thank the member companies of the PDL Consortium (including APC, EMC, Facebook, Google, HP Labs, Hitachi, IBM,
Intel, Microsoft Research, NEC Labs, NetApp, Oracle, Panasas, Riverbed, Samsung, Seagate, STEC, Symantec, and VMware) for their interest, insights, feedback, and support.

\section{REFERENCES}

[1] E. Achtert, C. Böhm, H.-P. Kriegel, P. Kröger, and A. Zimek. Robust, complete, and efficient correlation clustering. In SDM, USA, 2007.

[2] P. K. Agarwal and N. H. Mustafa. k-means projective clustering. In PODS, pages 155-165, Paris, France, 2004. ACM.

[3] C. Aggarwal and P. Yu. Redefining clustering for high-dimensional applications. IEEE TKDE, 14(2):210-225, 2002.

[4] C. C. Aggarwal, J. L. Wolf, P. S. Yu, C. Procopiuc, and J. S. Park. Fast algorithms for projected clustering. SIGMOD Rec., 28(2):61-72, 1999.

[5] R. Agrawal, J. Gehrke, D. Gunopulos, and P. Raghavan. Automatic subspace clustering of high dimensional data for data mining applications. SIGMOD Rec., 27(2):94-105, 1998.

[6] C. Böhm, K. Kailing, P. Kröger, and A. Zimek. Computing clusters of correlation connected objects. In SIGMOD, pages 455-466, NY, USA, 2004.

[7] H. Cheng, K. A. Hua, and K. Vu. Constrained locally weighted clustering. PVLDB, 1(1):90-101, 2008.

[8] R. L. F. Cordeiro, A. J. M. Traina, C. Faloutsos, and C. Traina Jr. Finding clusters in subspaces of very large, multi-dimensional datasets. In ICDE, pages 625-636, 2010.

[9] J. Dean and S. Ghemawat. Mapreduce: Simplified data processing on large clusters. OSDI, 2004.

[10] U. Fayyad. A data miner's story - getting to know the grand challenges. In Invited Innovation Talk, KDD, 2007: Slide 61. Available at: http://videolectures.net/kdd07_fayyad_dms/.

[11] U. Kang, C. Tsourakakis, A. P. Appel, C. Faloutsos, and J. Leskovec. Radius plots for mining tera-byte scale graphs: Algorithms, patterns, and observations. SDM, 2010.

[12] U. Kang, C. Tsourakakis, and C. Faloutsos. Pegasus: A peta-scale graph mining system - implementation and observations. ICDM, 2009.

[13] H.-P. Kriegel, P. Kröger, M. Renz, and S. Wurst. A generic framework for efficient subspace clustering of high-dimensional data. In ICDM, pages 250-257, USA, 2005.

[14] H.-P. Kriegel, P. Kröger, and A. Zimek. Clustering high-dimensional data: A survey on subspace clustering, pattern-based clustering, and correlation clustering. $A C M$ TKDD, 3(1):1-58, 2009.

[15] R. Lämmel. Google's mapreduce programming model revisited. Science of Computer Programming, 70:1-30, 2008.

[16] G. Moise and J. Sander. Finding non-redundant, statistically significant regions in high dimensional data: a novel approach to projected and subspace clustering. In $K D D$, pages 533-541, 2008.

[17] G. Moise, J. Sander, and M. Ester. Robust projected clustering. Knowl. Inf. Syst., 14(3):273-298, 2008.

[18] A. K. H. Tung, X. Xu, and B. C. Ooi. Curler: finding and visualizing nonlinear correlation clusters. In SIGMOD, pages 467-478, New York, NY, USA, 2005.

[19] M. L. Yiu and N. Mamoulis. Iterative projected clustering by subspace mining. IEEE TKDE, 17(2):176-189, Feb. 2005. 